\title{
The Effect of COVID-19 in Selecting Otolaryngology as a Specialty
}

\author{
Beatrice Go BS ${ }^{1}$ \\ ${ }^{1}$ University of Pennsylvania
}

April 27, 2020

\begin{abstract}
Coronavirus disease 2019 has undoubtedly impacted the healthcare system while causing lasting and profound implications for medical education. Senior medical students seeking exposure to the field of otolaryngology now find themselves in the challenging position of obtaining the experiences to make an informed decision on a future specialty. Virtual electives using comprehensive online material, discussion, and videos as well as the advent of telemedicine may be potential solutions to increase exposure to otolaryngology. While incorporating opportunities for authentic patient interactions is still a challenge, it is crucial that the academic otolaryngology community prioritize seeking solutions for interested medical students
\end{abstract}

\section{Beatrice Go BS ${ }^{1}$, Karthik Rajasekaran $\mathrm{MD}^{2}$}

${ }^{1}$ University of Pennsylvania, Perelman School of Medicine, Philadelphia, PA

${ }^{2}$ Department of Otorhinolaryngology, University of Pennsylvania, Philadelphia, PA

\section{Corresponding Author:}

Beatrice Go

800 Walnut St, $18^{\text {th }}$ Floor

Philadelphia PA 19107

Beatrice.go@pennmedicine.upenn.edu

The authors have no conflicts of interest, financial or other, to disclose.

Key Words: COVID-19 pandemic; medical education; medical student; residency decision making

\begin{abstract}
Coronavirus disease 2019 has undoubtedly impacted the healthcare system while causing lasting and profound implications for medical education. Senior medical students seeking exposure to the field of otolaryngology now find themselves in the challenging position of obtaining the experiences to make an informed decision on a future specialty. Virtual electives using comprehensive online material, discussion, and videos as well as the advent of telemedicine may be potential solutions to increase exposure to otolaryngology.
\end{abstract}


While incorporating opportunities for authentic patient interactions is still a challenge, it is crucial that the academic otolaryngology community prioritize seeking solutions for interested medical students

\section{Introduction}

The outbreak of coronavirus disease 2019 (COVID-19) originating from Wuhan, China has now rapidly transitioned into a global pandemic. The etiological agent of COVID-19, known as severe acute respiratory syndrome coronavirus 2 (SARS-CoV-2), has infected more than 2.3 million people worldwide causing 157,847 deaths, according to the World Health Organization (WHO) as of April 20, 2020. ${ }^{1}$ The United States alone currently makes up more than 745,000 of the infected patients, with numerous cases rising everyday. ${ }^{2}$ Given the high rates of transmission especially through asymptomatic carriers, many governing bodies and leaders have called for its citizens to stay at home to prevent overwhelming the healthcare system and its limited resources.

While COVID-19 has undoubtedly triggered serious economic losses, the pandemic will have profound and lasting implications on graduate medical education. University hospitals have had to take the unprecedented route of suspending classes and mandating all non-essential staff to stay at home. Though many medical students have mobilized to create organizations to support frontline workers, medical training is likely to suffer without emergent measures to provide continuing education. Particularly for those interested in a surgical field, it is challenging to develop a curriculum that is able to closely replicate the experience of the operating room. The situation is even more complex for students who are undecided on their specialty choice, as COVID-19 may affect desires to match into historically competitive fields, such as otolaryngology. We hope to emphasize how COVID-19 will impact exposure to otolaryngology and residency decision making, as well as propose solutions to mitigate disruption to medical education.

\section{Discussion}

In the standard 4-year medical school curriculum, the third year marks the transition from preclinical studies into the clinical environment full time. Medical students at this stage typically complete one year of core clinical rotations through a variety of fields such as internal medicine, general surgery, and pediatrics. In particular, the summer of third year is a crucial time to take additional electives, form meaningful mentoring relationships, and complete research endeavors before the residency application deadline. These several months are invaluable and allow undecided medical students to gather data and make an informed decision about their future specialty. A sub-internship during this time further increases exposure and allows for full immersion into the rigorous resident training environment. For competitive and specialized fields like otolaryngology, the above experiences are invaluable for the applicant to successfully match.

As a surgical subspecialty, otolaryngology electives are only pursued by a small minority of medical students per year. Physical restraints due to COVID-19 will further limit medical students from taking monthlong clinical electives in the field. And, while otolaryngology is an ever evolving and growing discipline, smaller institutions or those located in rural regions may not have a home otolaryngology program to allow for adequate exposure to the field. Students coming from such institutions may be disproportionately affected especially due to the travel restraints and social distancing precautions. Furthermore, away rotations at different institutions typically help students distinguish and choose their residency program based on preferred geographic location, program size, on-call responsibilities, teaching methods, and a sense of the whether a program is a good fit for them. Given the uncertainty and progression of the virus at the time of publication, it is plausible that such experiences may not occur during the 2020-2021 application cycle.

As a result of the pandemic, a significant number of students may not have the opportunity to learn more about otolaryngology. The ideal solution to promote student engagement and interest within the constraints of COVID-19 should include both the opportunity for scholarship and clinical application. The rapid emergence of telemedicine and teleteaching may hold the key to this solution. One of the advantages to videoconferencing is widespread accessibility and flexibility, allowing learners from distant institutions and locations to participate. Teaching techniques such as the flipped classroom approach or blended learning 
are easily applicable to videoconferencing and have been found to be effective. ${ }^{3-5}$ Students could potentially enroll in a structured virtual clinical elective. A curriculum could be created that includes selected readings and surgical videos from sources like the American Head and Neck Society, otolaryngology journals, and YouTube. An assigned attending can then discuss the videos and readings with the student. Students can also participate in resident didactics, grand rounds and tumor boards virtually. Suture materials can be distributed to allow students to practice knot tying and techniques, which can be reviewed by the attendings or residents. Students can also be given a research project that they can complete even after the rotation is finished. Lastly, opportunities for weekly mentorship meetings could be established, which could be an ideal platform for meaningful discussion and advice.

The obvious challenge and drawback is the inability to provide genuine patient interactions. Creating and using available virtual cases can promote critical thinking and increase clinical exposure, but there still remains a scarcity of opportunities for patient interaction especially in a surgical setting.

\section{Conclusion}

As healthcare systems and resources are being overextended during this time, it is inevitable that COVID-19 will also impact medical education and specialty decision making. The implications for a surgical subspecialty such as otolaryngology include reduced exposure and limited opportunities for medical students to learn and consequently, match into the field. Virtual electives and telemedicine are promising strategies to engage medical students, but it is crucial for the academic otolaryngology community to prioritize and develop practical solutions for continuing medical education.

\section{References}

1. World Health Organization. Coronavirus disease 2019 (COVID-19) Situation Report - 91. https://www.who.int/docs/defar source/coronaviruse/situation-reports/20200420-sitrep-91-covid-19.pdf?sfvrsn=fcf0670b_4. Accessed April $21,2020$.

2. Center for Disease Control. Cases in U.S. https://www.cdc.gov/coronavirus/2019-ncov/cases-updates/casesin-us.html. Accessed April 21, 2020.

3. Lin Y, Zhu Y, Chen C, et al. Facing the challenges in ophthalmology clerkship teaching: Is flipped classroom the answer? PLoS One . 2017;12(4):1-14. doi:10.1371/journal.pone.0174829

4. Hew KF, Lo CK. Flipped classroom improves student learning in health professions education: A metaanalysis. BMC Med Educ . 2018;18(1):1-12. doi:10.1186/s12909-018-1144-z

5. Milic NM, Trajkovic GZ, Bukumiric ZM, et al. Improving education in medical statistics: Implementing a blended learning model in the existing curriculum. PLoS One . 2016;11(2):1-10. doi:10.1371/journal.pone.0148882 